\title{
Research Paper \\ The Success Rate and Complications of Post-radiofrequency Ablation in Patients With Small Saphenous Insufficiency
}

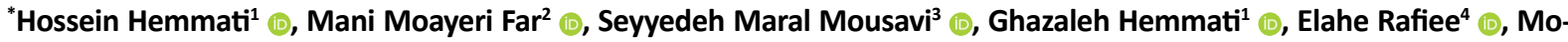 \\ hammad Taghi Ashoobi ${ }^{1}$ (C)
}

1. Department of General Surgery, Razi Clinical Research Development Unit, Guilan University of Medical Sciences, Rasht, Iran.

2. Department of Internal Medicine, Inflammatory Lung Diseases Research Center, Razi Hospital, School of Medicine, Guilan University of Medical Sciences, Rasht, Iran.

3. Department of Internal Medicine, Razi Clinical Research Development Unit, Guilan University of Medical Sciences, Rasht, Iran.

4. Razi Clinical Research Development Unit, Guilan University of Medical Sciences, Rasht, Iran.

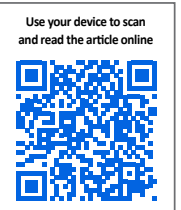

Chtrat Hemmati H, Moayeri Far M, Mousavi SM, Hemmati Gh, Rafiee E, Taghi Ashoobi M. [The Success Rate and Complications of Post-radiofrequency Ablation in Patients With Small Saphenous Insufficiency (Persian)]. Quarterly of "The Horizon of Medical Sciences". 2021; 27(3):368-383. https://doi.org/10.32598/hms.27.3.3376.1

dol'https://doi.org/10.32598/hms.27.3.3376.1

\section{AB STRACT}

Key words: Small saphenous vein, Radiofrequency, Varicose veins
Received: 06 Apr 2020 Accepted: 26 May 2021 Available Online: $01 \mathrm{Jul} 2021$

\section{Extended Abstract}

\section{Introduction}

aricose veins are abnormal dilation; the

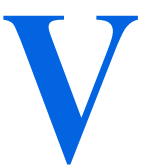
torsion of superficial veins is among the most common problems. The prevalence of varicose veins in the general population equals $10 \%$, i.e., higher than the total rate of coronary heart disease, peripheral
Lower extremity venous valve insufficiency affects $25 \%$ of women and $15 \%$ of men in the United States and Europe. Saphenous-Popliteal insufficiency and small saphenous vein reflux are less common than large saphenous vein reflux; however, they can lead to equally severe symptoms. Surgery for a small saphenous vein is also more challenging than a large one. The odds of potential damage to the sural nerve prevents numerous vascular surgeons from routinely performing small saphenous vein strapping [3, 4]. Additionally, the recurrence rate after small saphenous vein surgery in 5 years equals $30 \%-50 \%[5,6]$. Sclerotherapy is arterial disease, congestive heart failure, and stroke $[1,2]$.

\section{* Corresponding Author:}

Hossein Hemmati, MD.

Address: Department of General Surgery, Razi Clinical Research Development Unit, Guilan University of Medical Sciences, Rasht, Iran. Tel: +98 (911) 3331881

E-mail: drhossein.hemmati@gmail.com 
also a low-risk method for treating venous reflux; however, its failure rate for small saphenous veins with a diameter less than and more than $5 \mathrm{~mm}$ is estimated at $14 \%$ and $23 \%$, respectively [7].

Untreated varicose veins lead to pain, thrombophlebitis, bleeding, chronic venous insufficiency, and venous ulcers; thus, treatment has long been considered. Accordingly, various methods, including varicose sock, surgery, and more recently, minimally invasive methods were used to treat varicose veins [8]. Among these minimally invasive methods is using Radiofrequency Ablation (RFA) or Endovenous Laser Therapy (EVLT) of heat energy for the veins involved. These techniques are mostly used for treating large saphenous vein reflux as an alternative to surgical stripping [9]. In RFA, the heat generated by the catheters leads to heat damage to the venous wall, resulting in thrombosis and fibrosis. RF ablation is less invasive than surgical stripping; however, its potential complications include paresthesia, superficial thrombophlebitis, deep vein thrombosis, pulmonary embolism, bruising, skin burns, and infection [10].

The results of endovenous ablation techniques in treatment were at least equal to stripping; however, they were associated with reduced complications and enhanced Quality of Life (QoL) [11, 12]. Additionally, RFA and EVLT are associated with a concern about the recurrence of the disease in the form of recanalization and reflux; i.e., reached the same level as surgery due to technological advances, especially in new methods and some lasers. In contrast, other studies presented an improvement in QoL and a faster return to normal activity and work in ablation [13]. Almeida et al. reported that RFA was significantly better than EVL concerning post-procedure recovery and QoL parameters [14]. Rajagopala et al. stated that EVL and RF results were desirable and indicated few adverse effects [15].

Due to the high prevalence of the disease and further tendencies to minimally invasive interventions due to fewer recurrences and complications, it seems necessary to examine more closely and obtain information to improve the clinical decision-making process in similar cases. In this regard, this study aimed to determine the success rate and complications occurring after RFA in patients with small saphenous insufficiency who underwent RF ablation.

\section{Materials and Methods}

This case series was conducted on patients with varicose veins who were referred to a vascular surgery clinic in Rasht City, Iran, from 2009 to 2015. The study participants manifested small saphenous vein insufficiency with a diameter of $>4 \mathrm{~mm}$ and underwent RF ablation. Moreover, patients with varicose veins and small saphenous insufficiency with a diameter $<4 \mathrm{~mm}$ as well as a history of Deep Vein Thrombosis (DVT) who were not suitable candidates for RFA were excluded from this research.

In this clinic, the patients referring with varicose veins were examined for small saphenous vein insufficiency; if the small saphenous vein insufficiency was confirmed by sonography, the patients underwent RF ablation. RF ablation is a minimally invasive procedure. Accordingly, vascular endothelium is destroyed by a failed intravenous catheter with the help of radio waves (heat), causing thrombosis, fibrosis, and destruction. RF ablation is performed with a closure fast device in lying on stomach position under tumescent anesthesia. Simultaneously with this procedure, if necessary, the sclerotherapy of varicose veins was performed.

Then, the study patients were assessed concerning success rate (the absence of reflux in the first control ultrasound 2 weeks after RFA) as well as potential complications, such as postoperative pain, hematoma, paresthesia of the thigh and leg, neuropathy, Endovenous Heat Induced Thrombosis (EHIT), and skin burns. They were followed up 2 weeks after the procedure according to the treatment protocol. The study patients were examined for the presence or absence of hematoma and asked about paresthesia of the legs and thighs.

Reanalysis was also evaluated in the research patients by examination and ultrasound at 2 weeks, 3-6 months, and one year after RFA by a sonographer. If there existed clinical and sonographic evidence of DVT as a procedure complication, it was classified according to the EHIT classification [10].

Further data, including age, gender, Body Mass Index (BMI), occupation, the degree of varicose veins based on Clinical-Etiological-Anatomical-Pathophysiological (CEAP) score [16], potential complications, such as postoperative pain based on VAS (visual analog scale), hematoma, thigh and leg paresthesia, neuropathy, EHIT, skin burns, reanalysis, and reflux were extracted from the study patients' medical records. According to heparin use after 2013 during RFA, the research patients were divided into two groups before 2013 (without heparin use ), and after 2013 (heparin use).

Relevant data were collected through data collection forms and analyzed using SPSS. To report the obtained results, frequency and percentage were used for qualitative variables. Besides, mean and standard deviation, mean (mid-quarter range), and minimum-maximum indices were employed for quantitative variables. The normal distributions of the study quantitative variables were measured us- 
ing the Shapiro-Wilk's test. The Wilcoxon test was used to compare the degree of varicose veins and VCSS score [17] before and after RF ablation. Furthermore, comparisons between complications and variables (age, gender, BMI, occupation) were performed using the t-test and Fisher's Exact test. The significance level of the tests was considered $\mathrm{P}<0.05$.

\section{Results}

In total, 62 patients with varicose veins and small saphenous vein insufficiency who underwent RF ablation were studied. The Mean \pm SD age of the examined patients was $51.50 \pm 12.12$ years. Moreover, $54.8 \%$ of the study patients were male and the rest were female. Occupationally, the highest frequency was related to housewives $(41.9 \%)$, followed by employees (37.1\%), and freelancers (19.4\%). The study subjects' Mean \pm SD BMI was measured to be $26.49 \pm 1.45 \mathrm{~kg} / \mathrm{m}^{2}$.

Based on the obtained data, the success rate or absence of reflux in the first control ultrasound 2 weeks after RFA equaled $100 \%$; no case of reflux was observed in the first control ultrasound 2 weeks after RF ablation. The highest frequency of varicose veins before and after RF ablation concerned C2 (69.4\%) and C1 (56.5\%), respectively (Table 1).

In general, $f$ the Wilcoxon test data indicated a significant difference between the degree of varicose veins before and after $\mathrm{RF}$ ablation $(\mathrm{P}<0.001)$. Thus, in 48 patients, the degree of varicose veins decreased, compared to before RF ablation, and increased in one case. It also remained unchanged in 13 cases. Additionally, the results of venous clinical severity score before and after RF ablation revealed that the VCSS score after RF ablation significantly decreased $(\mathrm{P}<0.001)$, compared to before (Table 2).

The Mean \pm SD pain score after RF ablation was calculated as $3.03 \pm 2.04$ (median: 3 ; range: $0-8$ ). The results of treatment complications at 2 weeks, 3-6 months, and the first year after RF ablation indicated that the most complications in 2 weeks after RF ablation included neuropathy $(14.5 \%)$ and heat thrombosis $(11.3 \%)$, respectively. No recanalization was observed 2 weeks after RF ablation. In 3-6 months, 2 cases of recanalization were observed; only in one of them, recanalization was detected in the first year. Furthermore, in the first year after RF ablation, a case of recanalization was observed.

In 2 weeks after RF ablation, 7 cases of heat thrombosis were observed ( 3 cases of second degree, 2 cases of first degree, 1 case of third-degree, \& 1 case of 4 th degree). Of these, heat thrombosis was observed in 3 patients at 3-6 months' follow-up (first-, second-, \& fourth-degree). No case of heat thrombosis was observed in the first year after RF ablation.

In 2 weeks after RF ablation, 9 cases of neuropathy were observed. Neuropathy was not detected in any of these 9 patients in the subsequent follow-ups. In the 3-6 months and the first year after RF ablation, no new cases of neuropathy existed in the study subjects.

At 2 weeks, 3-6 months, and the first year after RF ablation, no cases of skin burns were observed in the examined subjects. Furthermore, in 3-6 months after RF ablation, only 1 case of superficial thrombophlebitis was observed. No cases of superficial thrombophlebitis were observed between 2 weeks and the first year after RF ablation.

The most common complication in 2 weeks after RF ablation was neuropathy $(14.5 \%)$, followed by heat thrombosis (11.3\%). Based on the results of the t-test and Fisher's Exact test, there was no significant relationship between heat thrombosis and neuropathy in two weeks after RF ablation, and demographic variables (age, gender, BMI, \& occupation) $(\mathrm{P}>0.05)$.

Moreover, according to Fisher's Exact test findings, there was no significant relationship between heat thrombosis $(\mathrm{P}=0.648)$ and neuropathy $(\mathrm{P}=0.238)$ two weeks after $\mathrm{RF}$ ablation, and the degree of varicose veins before RF ablation.

According to the results of the Fisher's Exact test, there was no significant relationship between heat thrombosis $(\mathrm{P}>0.999)$ and neuropathy $(\mathrm{P}=0.242)$ two weeks after RF ablation and the year of its occurrence (before \& after 2013) (Table 3).

\section{Discussion}

This study determined the success rate and complications after RFA in patients with small saphenous insufficiency who underwent RF ablation in a vascular surgery clinic from 2009 to 2015. In total, 62 patients with varicose veins who had small saphenous vein insufficiency and underwent $\mathrm{RF}$ ablation were studied.

The Mean \pm SD age of the research subjects was $51.5 \pm 12.12$ years with a median of 54 years. The minimum and maximum age of the study participants were 24 and 76 years, respectively. Furthermore, $54.8 \%$ of the explored patients were male and the rest were female. In general, in different studies, the age group and the gender distribution of patients were similar to those of the present study [18-20]. Occupationally, the highest frequency orderly concerned 
Table 1. The frequency of varicose veins before and after RF ablation by varicose veins

\begin{tabular}{|c|c|c|c|}
\hline \multirow{2}{*}{ Varicose Veins (CEAP) } & \multicolumn{3}{|c|}{ No. (\%) } \\
\hline & Before RF Ablation & $\begin{array}{c}\text { Varicose Vein Changes (CEAP) } \\
\text { After RF Ablation }\end{array}$ & After RF Ablation \\
\hline $\mathrm{C} 1$ & 0 & 0 & $35(56.5)$ \\
\hline $\mathrm{C} 2$ & $43(69.4)$ & $\begin{array}{l}\text { grade } \mathrm{C} 132(74.4) \\
\text { grade } \mathrm{C} 210(23.3) \\
\text { grade C3 } 1(2.3)\end{array}$ & 19(30.6) \\
\hline C3 & $5(8.1)$ & $\begin{array}{l}\text { C2 3(60) } \\
\text { C1 2(40) }\end{array}$ & $2(3.2 \%)$ \\
\hline $\mathrm{C} 4$ & 11(17.7) & $\begin{array}{l}\text { C2 6(5.54) } \\
\text { C4 (3.27) } \\
\text { C3 1(1.9) }\end{array}$ & $6(9.7 \%)$ \\
\hline C5 & $3(4.8)$ & C1 1(1.9) & 0 \\
\hline
\end{tabular}

housewives (41.9\%), employees (37.1\%), and freelancers (19.4\%). The study subjects' mean \pm SD BMI was measured as $26.49 \pm 1.45 \mathrm{~kg} / \mathrm{m}^{2}$. In the study of Nehemiah Samuel et al., the same rate was equal to $25.9 \pm 3.2 \mathrm{~kg} / \mathrm{m}^{2}$, i.e., almost similar to ours [21]; no significant difference could be considered for it. In the present study, the highest frequency respected the degree of $\mathrm{C} 2$ varicose veins, i.e., consistent with the study of Jung Hyun Choi and associates [20]. This alignment was also observed in the study of Nehemiah Samuel [21], S. Doganci [22], L van Groenendael [23], and Margreet Trip-Hoving [24].

The success rate in our study was computed to be $100 \%$; no case of reflux was observed in the first control ultrasound 2 weeks after RF ablation. This rate equaled 100\% in the study of Thomas S. Monahan et al. [18], i.e., consistent with the data obtained by Boersma D et al. (97.1\%) [25], and Nehemiah Samuel et al. (96.2\%) [21] in 3 months. Michael Harlander-Locke et al., in a $24-72$ hours after RF ablation follow-up, suggested that in $98.7 \%$ of the cases, duplex ultrasound findings suggested the closure of the treated SSV fragment. In one patient, after 211 days of ablation, the treated fragment was open and did not consent to further operations [26]. Therefore, based on the results of the present study and similar studies, the success rate of this treatment method can be expected.

The Mean \pm SD pain score after RF ablation was calculated to be 3.03 \pm 2.04 ; however, in Margreet Trip-Hoving's study, this rate equaled 5.80 \pm 1.86 [24]; in Doganci et al.'s study, the duration of treatment was on average 1.2 days [22]. The data discrepancy may not be significant; however, the slight difference can also be explained by variations in the location of the study and the relative expression of pain using the Visual Analogue Scale (VAS).

In the present study, the post-treatment VCSS score of patients was significantly reduced, compared to pre-treatment, i.e., comparable with the data achieved by N.S. Theivacumar and colleagues. Higher VCSS levels at the end of treatment in this study may be due to greater pre-treatment

Table 2. VCSS score before and after RF ablation

\begin{tabular}{cccccc}
\hline VCSS score & Mean \pm SD & Mean(mid-quarter range) & Min. & Max. & P* \\
\hline Before & $5.53 \pm 1.91$ & $5(2.25)$ & 3 & 13 & $<0.001$ \\
After & $3.40 \pm 2.01$ & $3(2)$ & 1 & 11 & Quarterly of \\
* Wilcoxon test. & & & The Horizon of Medical Sciences
\end{tabular}


Table 3. The distribution of complications 2 weeks, 3-6 months, and the first year after RF ablation in patients with small saphenous vein insufficiency, before 2013 and after 2013

\begin{tabular}{cccccc}
\hline \multirow{2}{*}{$\begin{array}{c}\text { Duration after RF } \\
\text { Ablation }\end{array}$} & Year & \multicolumn{5}{c}{ No. (\%) } \\
\cline { 3 - 6 } 2 weeks & Before $2013(n=17)$ & Recanalization & Heat Thrombosis & Neuropathy & $\begin{array}{c}\text { Superficial Throm- } \\
\text { bophlebitis }\end{array}$ \\
\hline \multirow{2}{*}{3 3-6 months } & 2013 and above $(n=45)$ & - & $2(11.8)$ & $4(23.5)$ & - \\
& Before $2013(n=17)$ & - & $5(11.1)$ & $5(11.1)$ & - \\
First year & 2013 and above $(n=45)$ & $2(4.8)$ & - & - & - \\
& Before $2013(n=17)$ & - & - & - & - \\
\hline
\end{tabular}

VCSS; these data may also justify a further reduction in VCSS during treatment in this study [19].

In the present study, the incidence of heat thrombosis was $7(11.3 \%)$ cases; only $2(3.2 \%)$ of these 7 cases were graded as 4; however, in the studies of Theivacumar [19], Groenendael [23], D’ Othée [27], Doganci [22], Carradice [28], and Desmyttere [29] zero incidences of thrombosis was reported. Additionally, Boersma et al. documented an overall incidence of $0 \%-1.2 \%$ [25], i.e., significantly less than that in the present study. This fining can be primarily attributed to different definitions and how it is measured. All the above studies examined the presence of DVT; we only observed two cases of DVT. In the present study, intravascular heat thrombosis was explored. According to Lawrence et al., it has 4 degrees; grade 4 includes DVT [30]. Thus, the present study data indicate the significance of lower degrees of EHIT, i.e., disregarded in other studies. Moreover, the above studies, unlike the present study, investigated the effects of the laser ablation method. However, in the study of Marsh et al., the incidence of EHIT after radiofrequency ablation was reported to be approximately $98 \%$. The difference may be due to RFA in the present study on the small saphenous vein and the Marsh study of the effect of this method on the large saphenous vein [31]. Furthermore, ultrasound confirmation is required to diagnose EHIT, necessitating ultrasound by a qualified radiologist. Heparin prophylaxis is recommended to prevent EHIT.

The incidence of neuropathy in the present study was measured as $14.5 \%$; in Boersma's study, this rate equaled $4.7 \%$ and in Theivacumar's study, it was $4.4 \%$ [19]; i.e., significantly lower than the amount obtained in the present study. Samuel et al. reported the incidence of neurological problems to be $7.5 \%$ [21], i.e., about half of the statistics reported in the present study. This finding seems to be the source of this difference in the method of ablation. In the mentioned study and the present research, i.e., performed by radiofrequency method, this difference can also be explained by the underlying disease in older individuals, like those with diabetic neuropathy, and the proximity of the procedure to the sural nerve.

In our study, no recanalization was observed in 2 weeks after RF ablation. In 3-6 months and the first year after RF ablation, 2 cases of recanalization were observed (a common case between 3-6 months \& the first year). In the study of Nehemiah Samuel et al., 2 cases of recanalization were reported [21]. Margreet Trip-Hoving et al. also stated that the incidence of recanalization equaled $0 \%-4 \%$ [24]. Margreet Trip-Hoving et al. reported a recanalization incidence rate of $0 \%-4 \%$, i.e., a reasonable and citable limit. Additionally, in the present study, the rate of recanalization was 1 case per 6 months and 3 cases in a year, i.e., approximately $4 \%$ of the sample size. However, Ravi et al. stated this rate as $9 \%$ [15]; Groenendael et al reported a rate of $45 \%$ [23], suggesting a wide range of results. However, our data presented no significant relationship between this wide range of recanalization results. The current study results revealed no significant difference in using heparin after 2013, compared to before 2013; such a lack of significance could be due to the small sample size in this research.

\section{Conclusion}

Based on the obtained results, RFA treatment of small saphenous is a highly suitable and applicable method due to its high success rate and limited adverse effects. 


\section{Ethical Considerations}

\section{Compliance with ethical guidelines}

The Ethics Committee of Guilan University of Medical Sciences approved this study (Code: IR.GUMS. REC.1394.424).

\section{Funding}

This research did not receive any grant from funding agencies in the public, commercial, or non-profit sectors.

\section{Authors' contributions}

Conceptualization, supervision and methodology: Hossein Hemmati, Seyedeh Maral Mousavi and Ghazaleh Hemmati; Data collection: Mani Moayerifar and Mohammad Taghi Ashoubi; Data analysis: Elahe Rafiei; Investigation, writing - original draft, and writing - review \& editing: All authors.

\section{Conflicts of interest}

The authors declared no conflicts of interest.

\section{Acknowledgements}

The authors appreciate Gilan University of Medical Sciences and Razi Clinical Research Development Unit for facilitating the research process. 
This Page Intentionally Left Blank 


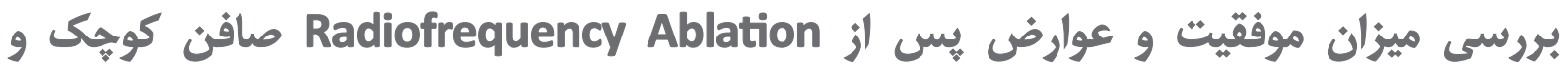

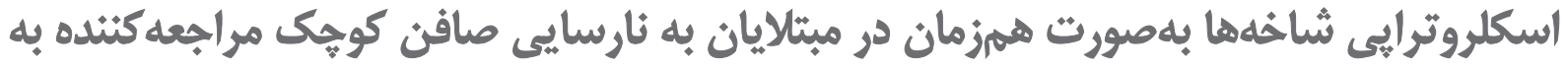

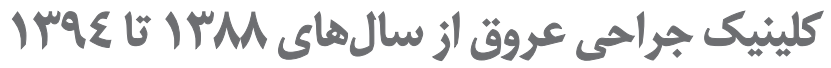

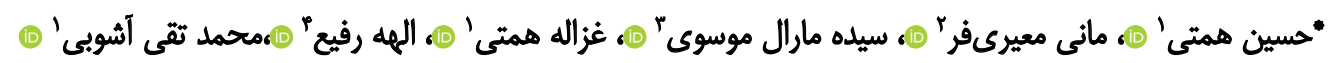 \\ ا.كروه جراحى عمومى، واحد توسعه تحقيقات بالينى رازى، دانشاه علوم يزشكى كيلان، رشت، ايران.

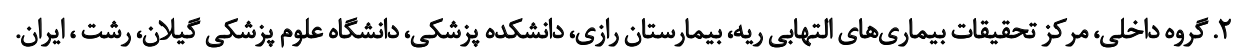

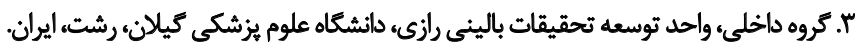

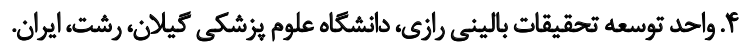

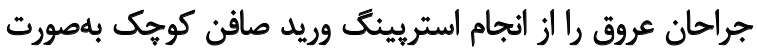

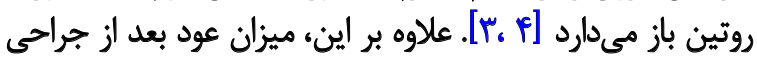

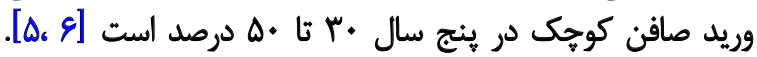

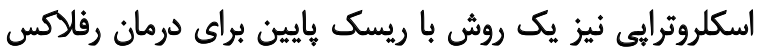

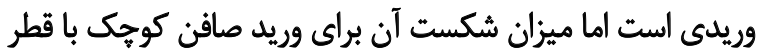

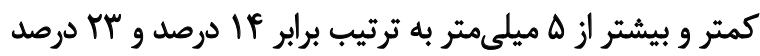

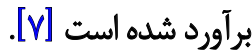
از آنجا كه درمان نكردن واريس منجر به درد، ترومبوفلبيت،
واريس نوعى اتساع غيرعادى و بيجخوردمى وريدهاى سطحى

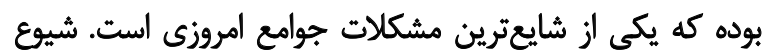

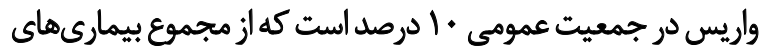

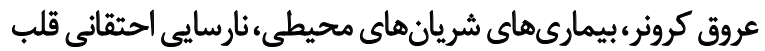

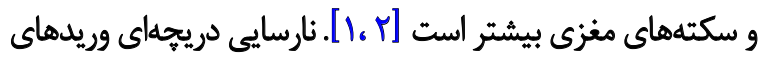

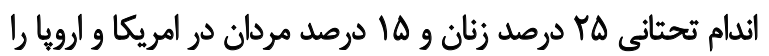

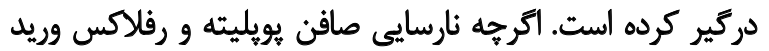

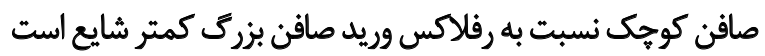

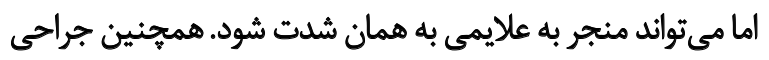


ترومبوز وريد عمقى (DVT) كه كانديد مناسب براى RFA نبودند،

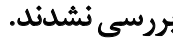

در اين كلينيك، بيماران واريسى مراجعهكنيده از نظر ابتلا

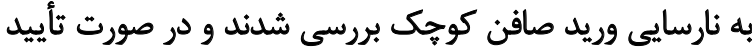

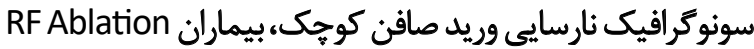

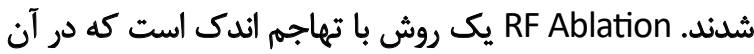
بله با كاتترى درون وريد نارسا و به كمك امواج راديويى (ترماييى)

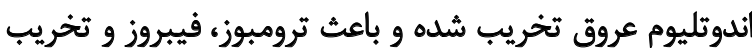

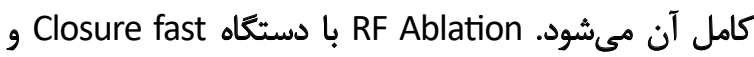

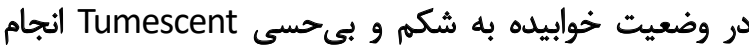

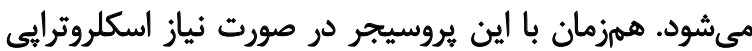

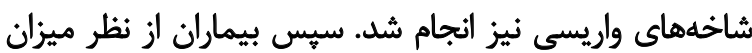

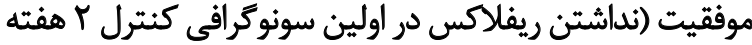

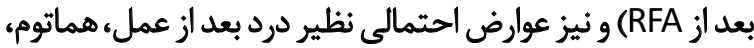

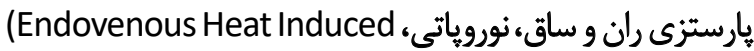

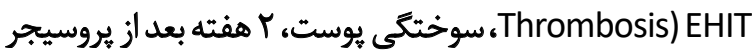

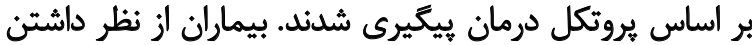

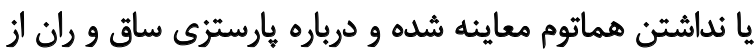

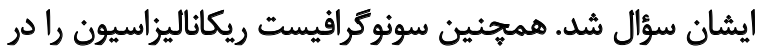

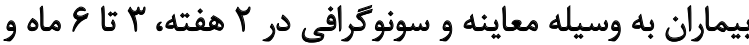

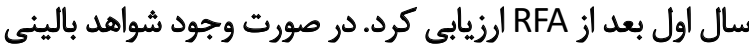

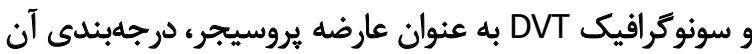

طبق EHIT Classification صورت كرفت [1.] متغيرهاى مطالعه كه شامل سن، جنس، BMI، شغل، درجه

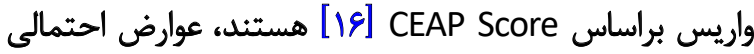

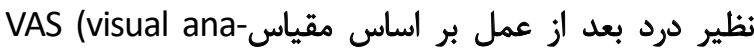

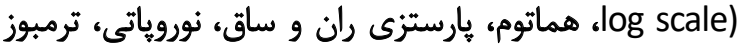

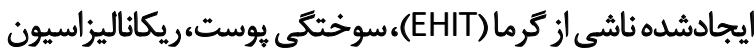

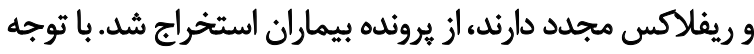

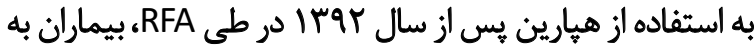

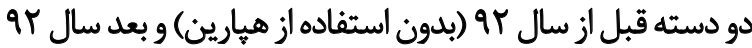

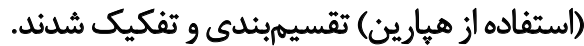

دادهاى مربوط بـ از جمع آورى از طريق فرمهاى اطلاعانى

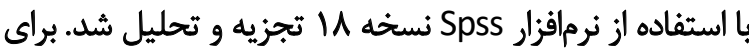

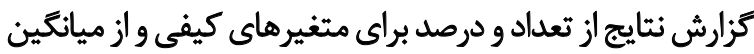

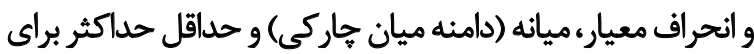

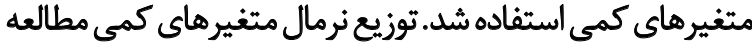

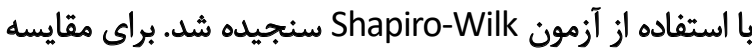

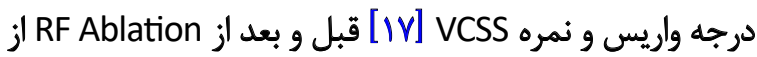

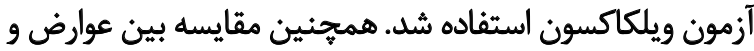

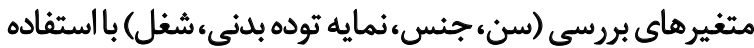

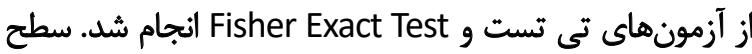

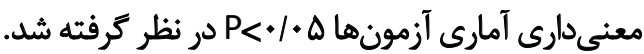

خونريزى، نارسايى مزمن وريدى و اولسرهاي وريدى مى مشود،

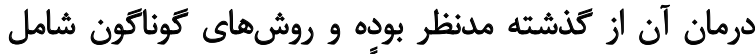

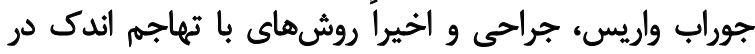

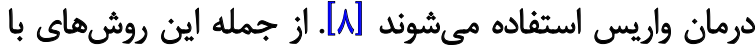

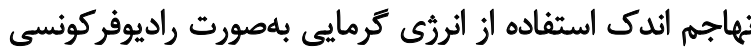
يا ليزر درمانى (RFA)

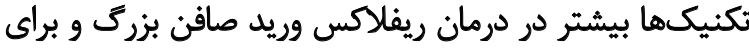

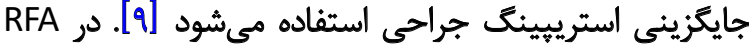

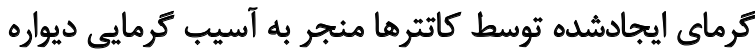

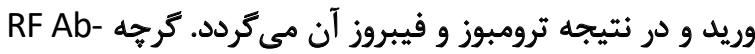

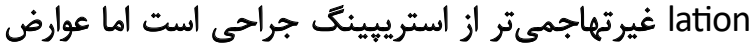

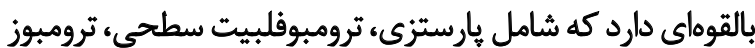

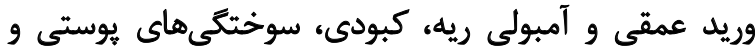

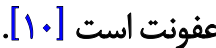

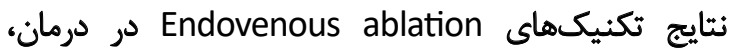

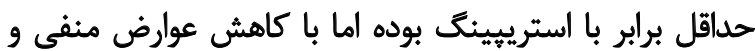

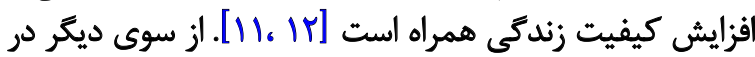

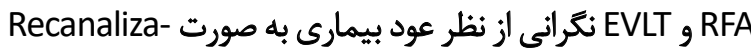

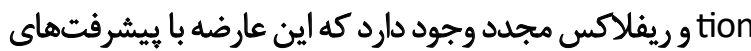

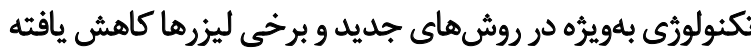

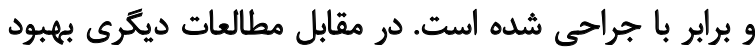

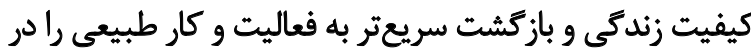

Ablation نشان دادند [1 [1]].

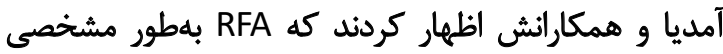

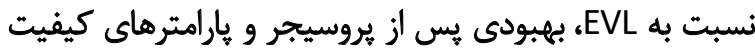

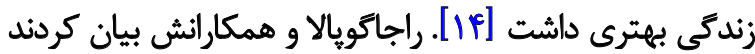

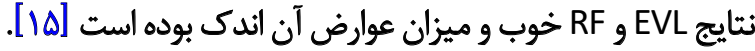
به دليل شيوع بالاى بيمارى و ترايشهاى بيشتر به روشهاى كاي

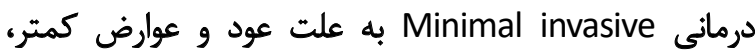

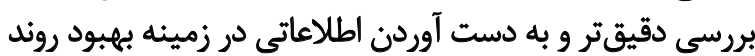

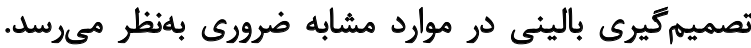

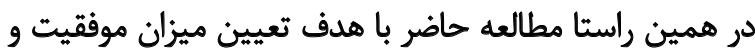

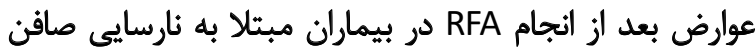

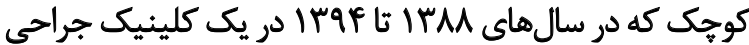
عروق RF Ablation شدند، انجام شد.

$$
\text { مواد و روشها }
$$

اين مطالعه به صورت كزارش موارد (Case Series) در بيماران

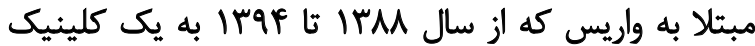

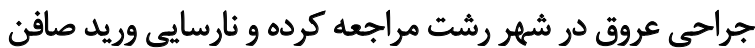

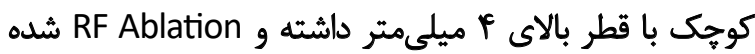

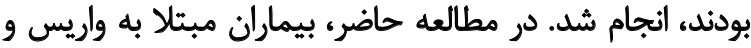

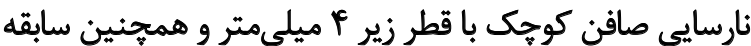




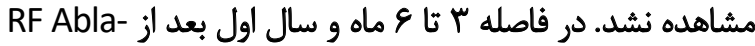

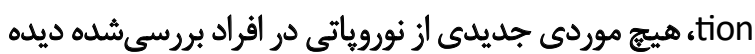

در فواصل r هفته، ب تا ع ماه و سال اول بعد از RF Ablation،

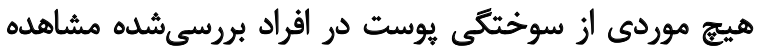

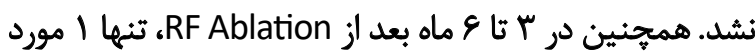

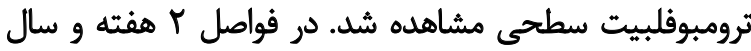

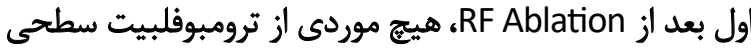

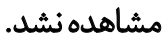

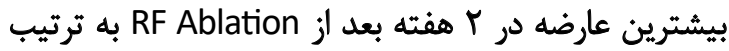

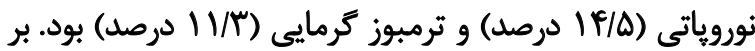

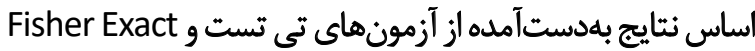

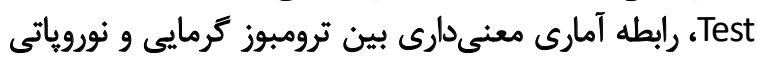

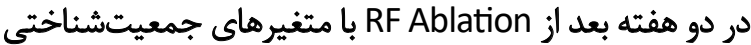

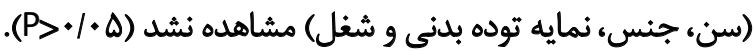

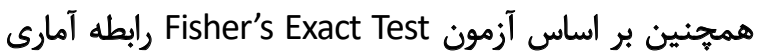

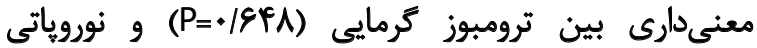

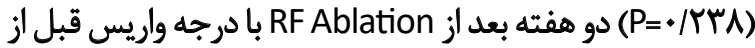

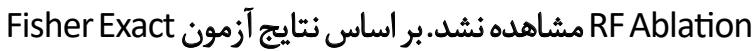

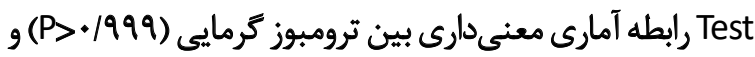

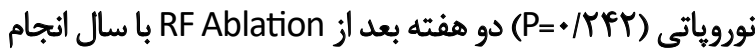

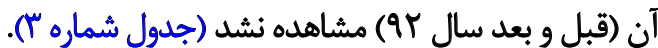

ث

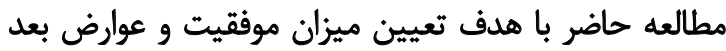

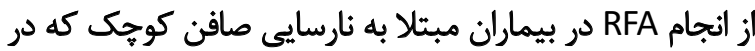

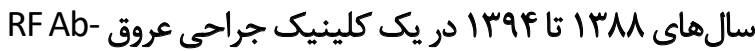
lation كه داراى نارسايى وريد صافن كوجى بون بوده و RF Ablation شده بودند، بررسى شند.

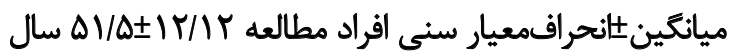

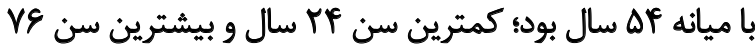

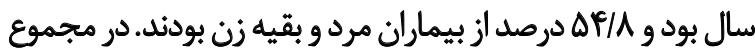

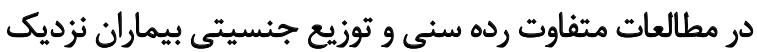

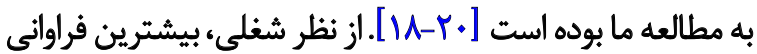

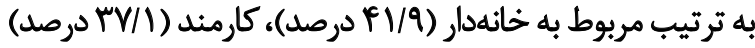

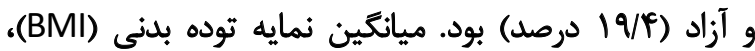

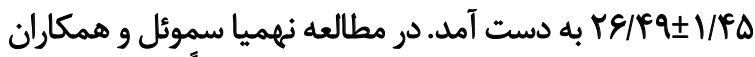

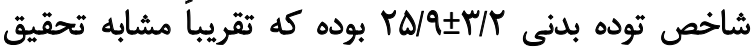
ماست [Mاخ و اختلاف معنادارى براى آن نمى توانوان در نظر كرفت.

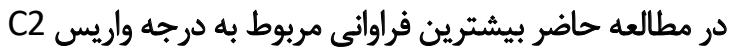

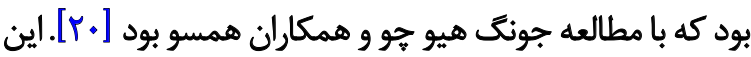

ياقتهها

در اين مطالعه بو بو بيمار مبتلا به واريس كه داراي نارسايي

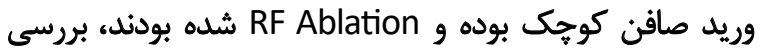

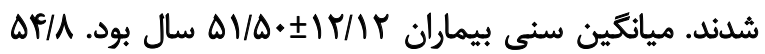

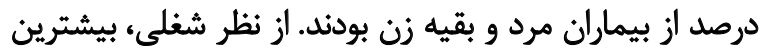

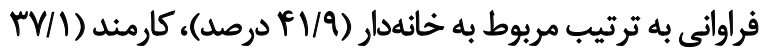

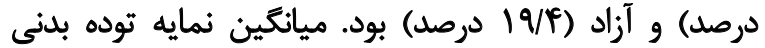
(BMI)

بر اساس نتايج بهدست آمده، ميزان موفقيت يا به عبارتى أز بين

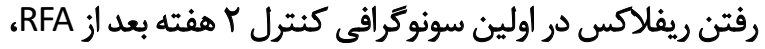

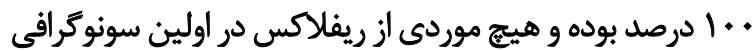

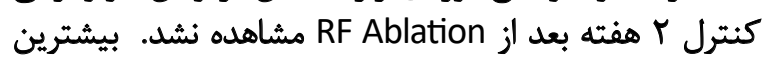

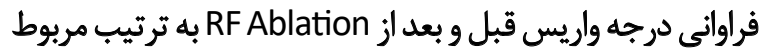

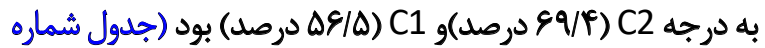

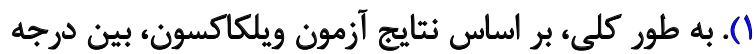

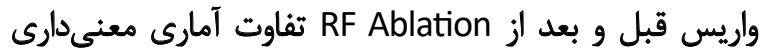

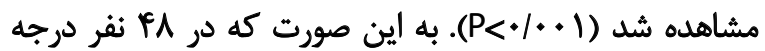

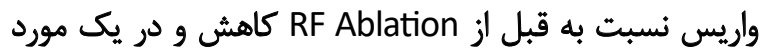
افزايش داشت، همجنين در به ال مورد بدون تغيير بود. نتايج بررسى نمره VCSS قبل و بعد از RF

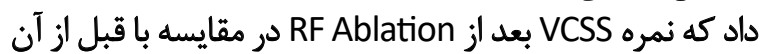

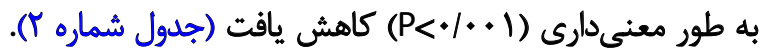

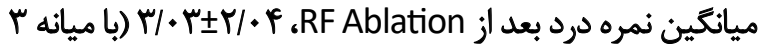

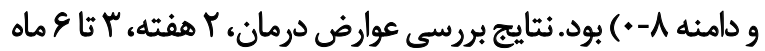

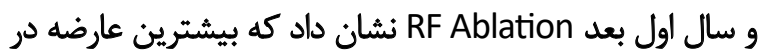

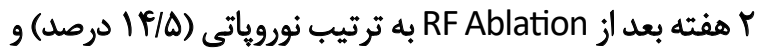

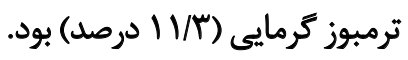

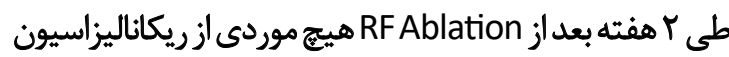

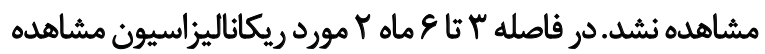

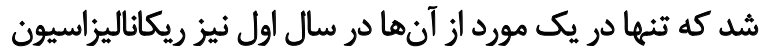

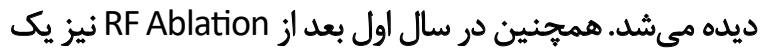
مورد ريكاناليزاسيون ديده شد. همجن

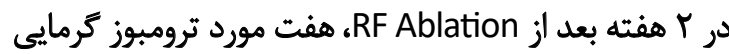

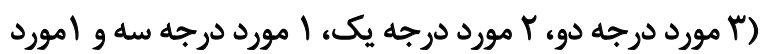

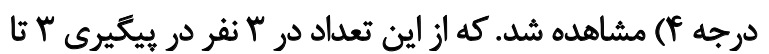

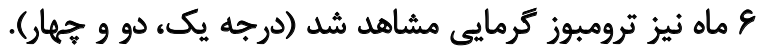

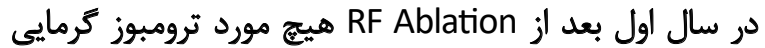

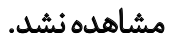
در Y هفته بعد از RF Ablation، ج و مورد نوروياتى مشاهده

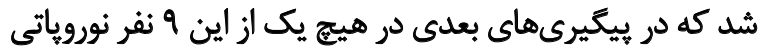

1. Venous Clinical Severity score 
جدول ا. فراوانى درجه واريس قبل و بعد از RF Ablation به تفكيك درجه واريس

\begin{tabular}{|c|c|c|c|}
\hline \multicolumn{3}{|c|}{ تعداد (درصد) } & \multirow{2}{*}{ ورجه واريس (CEAP) } \\
\hline بعد از RF ablation & تغييرات درجه واريس (CEAP) بعد از RF ablation) & قبل از RF ablation & \\
\hline$r \Delta(\Delta</ \Delta)$ & • & - & $\mathrm{C} 1$ \\
\hline \multirow{3}{*}{$19(\% \cdot 18)$} & 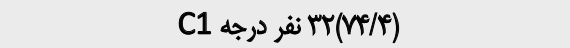 & \multirow{3}{*}{$P r(E q / F)$} & \multirow{3}{*}{$\mathrm{C} 2$} \\
\hline & (T/س/M)• نفر درجه C2 & & \\
\hline & C3 آنقر درجه (T/T) & & \\
\hline \multirow{2}{*}{$r(\Psi / T)$} & 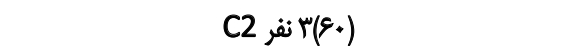 & \multirow{2}{*}{$\Delta(\mathbb{N})$} & \multirow{2}{*}{$\mathrm{C} 3$} \\
\hline & 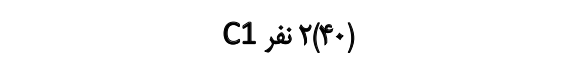 & & \\
\hline \multirow{4}{*}{$g(q / V)$} & 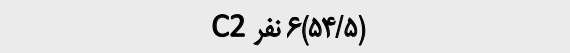 & \multirow{4}{*}{$11(I V / V)$} & \multirow{4}{*}{$\mathrm{C} 4$} \\
\hline & C4 نفر T/T/T) & & \\
\hline & 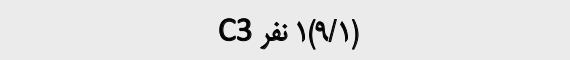 & & \\
\hline & 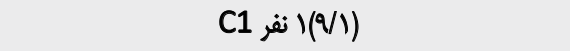 & & \\
\hline - & 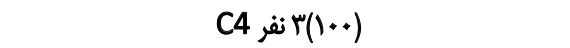 & $r(\% / A)$ & $\mathrm{C} 5$ \\
\hline
\end{tabular}

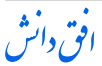

بود [YF]. در مطالعه دوكانسى و همكاران مدتزمان مران مورد نياز

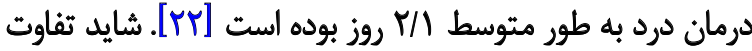

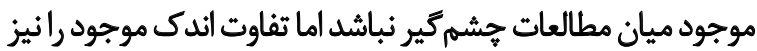

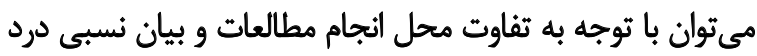

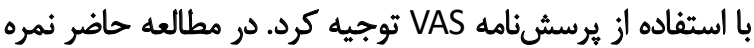

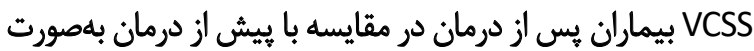

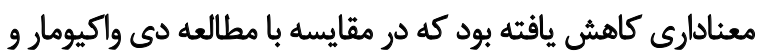

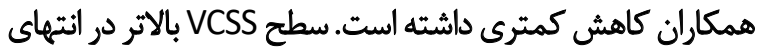

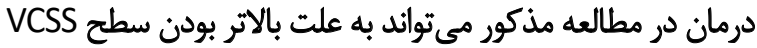

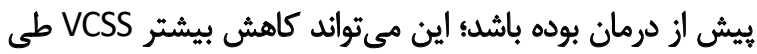

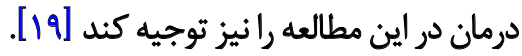

در مطالعه حاضر ميزان بروز ترومبوز گرمايى ل مورد (T/T)

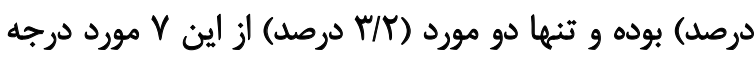

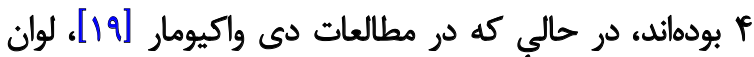

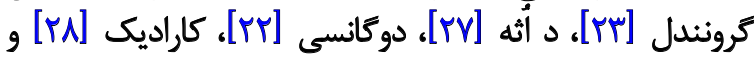

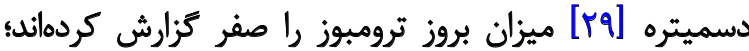

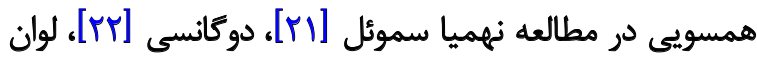

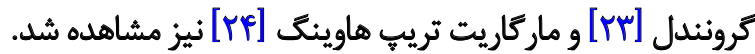
ميزان موفقيت در مطالعه ما ـ . ا درصد بوده و هيج موردى از

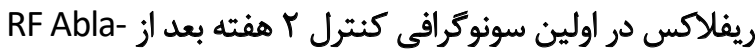

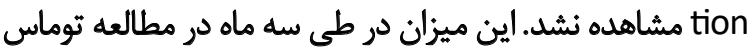

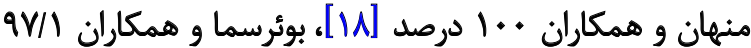

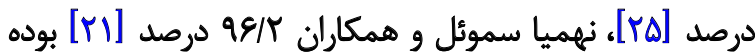

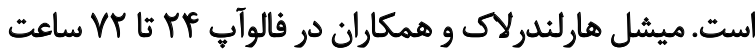

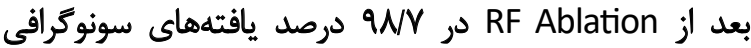

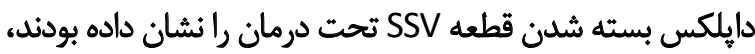

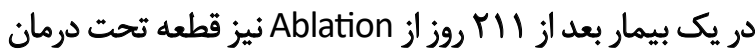

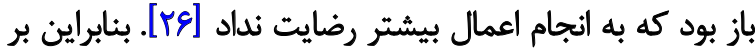

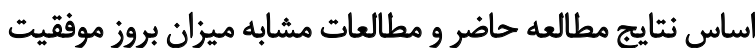

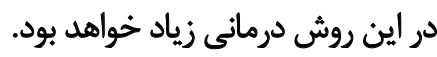

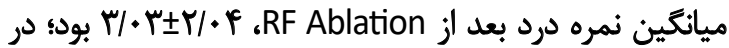

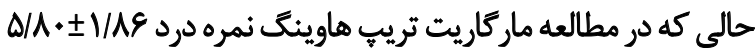

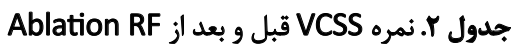

\begin{tabular}{|c|c|c|c|c|c|}
\hline P* & هداكثر & جداقل & ميانه (دامنه ميان هاركىى) & ميانكين ث|نحراف معيار & Vمره VCSS \\
\hline \multirow{2}{*}{$>\cdot 1 \cdot .1$} & ir & $r$ & $\Delta(r / T \Delta)$ & $\Delta / \Delta r \pm 1 / 91$ & قبل \\
\hline & 11 & 1 & $r(r)$ & $r / r+ \pm r / * 1$ & بعد \\
\hline
\end{tabular}




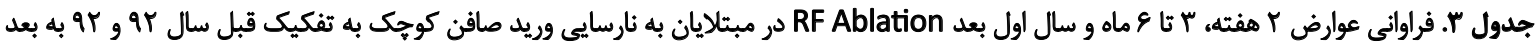

\begin{tabular}{|c|c|c|c|c|c|}
\hline \multicolumn{4}{|c|}{ تعداد (درصد) } & \multirow{2}{*}{ سال } & \multirow{2}{*}{ مدت زمان بعد از RF ablation } \\
\hline ترومبوفلييت سطحى & ثوروياتى & ترومبوز كرمايي & ريكاناليزاسيون & & \\
\hline- & $P(r T / Q)$ & $r(1 / / 1)$ & - & قبل Yra (n=IV) & \\
\hline- & $\Delta(11 / 1)$ & $\Delta(11 / 1)$ & - & rqو وبالاتر (n=ra & dies $Y$ \\
\hline- & - & - & - & قبل ra & \\
\hline$I(T / F)$ & - & - & $r(f / \Lambda)$ & ra & wer of \\
\hline- & - & - & - & قبل & \\
\hline- & - & - & $I(T / \Lambda)$ & ra و بالاتر (qس-r) & 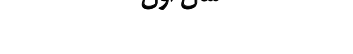 \\
\hline
\end{tabular}

أنّان

مطالعه حاضر به روش راديوفركوئنسي انجام شده است. همجنين

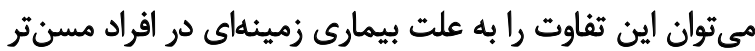

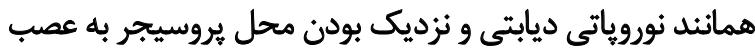
سورال توجيه كرد.

در مطالعه ما در Y هفته بعد از RF Ablation هيج موردى از ماز

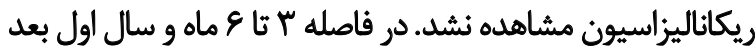

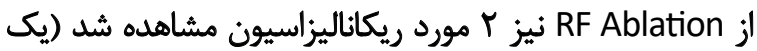

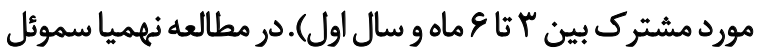

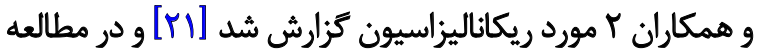

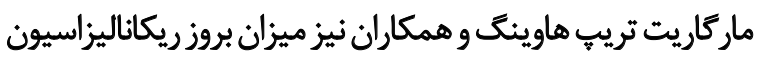

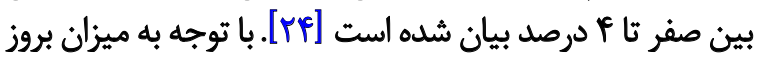

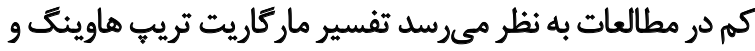

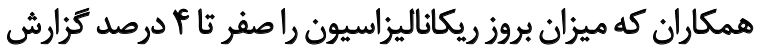

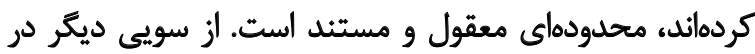

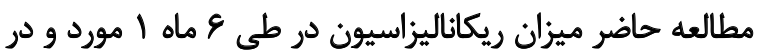

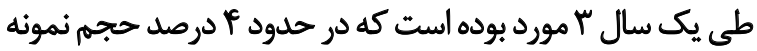

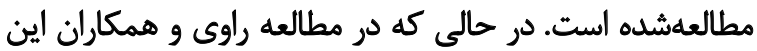
ميزان 9 درصد [1ه]

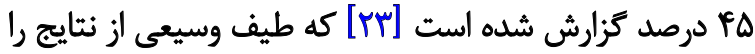

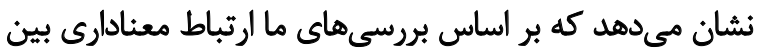

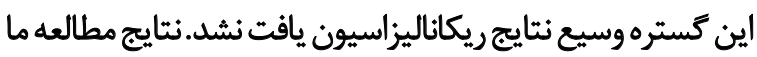

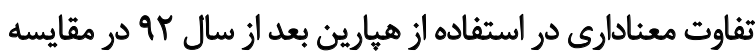

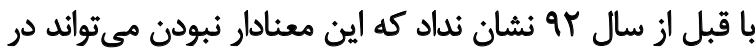

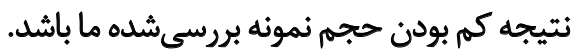

\section{تثيجِ}

بر اساس نتايج به دست آمله به نظر مىرسد درمان -Radio frequency Ablation زياد و عوارض اندك، روشى بسيار مناسب و اجرايى است.
همجنين بوئرسما و همكاران در مطالعه خود ميزان بروز كلى دئ

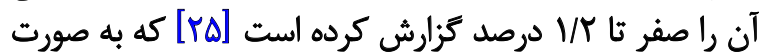

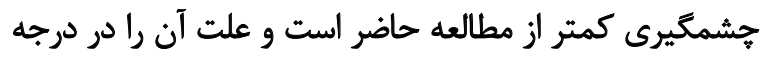

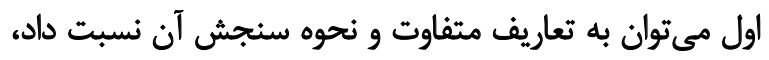

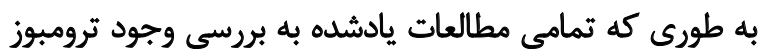

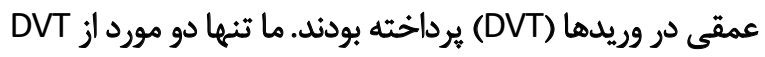

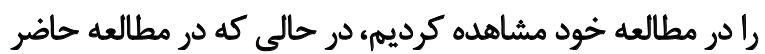

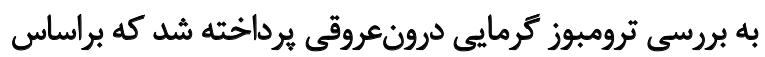

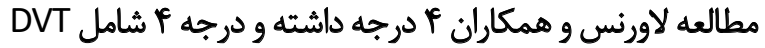

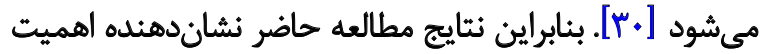

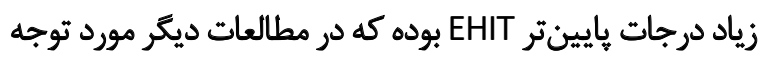

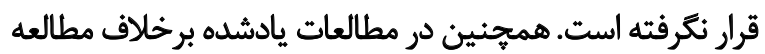

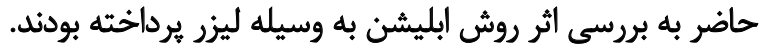

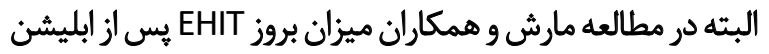

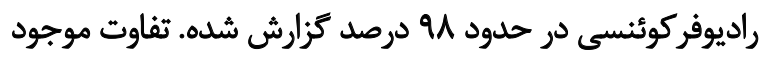

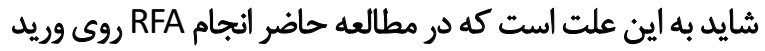

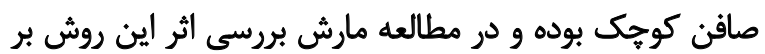

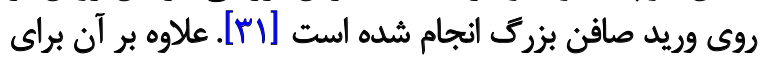
تشخيص EHIT نياز به تأييد سونوكرافى بوده؛ برائ اين كائ كار نياز

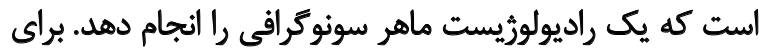

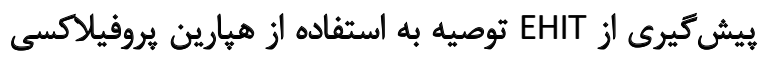

$$
\text { شده است. }
$$

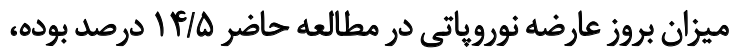

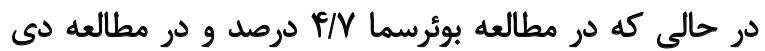

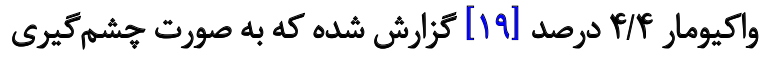

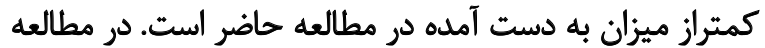

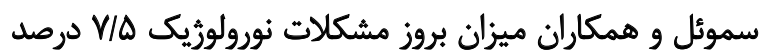

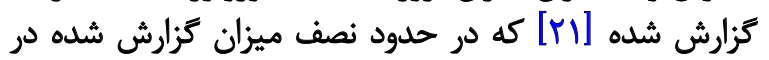

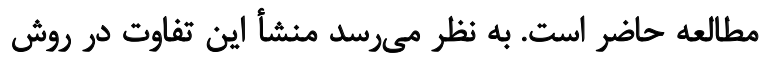

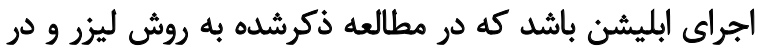


مالاحظات اخلاقى

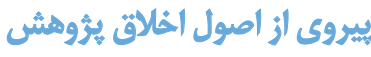

كميته اخلاق دانشعاه علوم ئششكى كيلان اين مطالعه را با كد اخلاق (IR.GUMS.REC.1394.424) تأييد نمود.

$$
\text { مامي مالي }
$$

اين تحقيق هيج كونه كمك مالى از سازمان هاى تأمين مالى در بخش هاى عمومى ، تجارى يا غيرانتفاعى دريافت نكرد.

$$
\text { مشاركت نويسندكّان }
$$

ارائه ايده ثيزوهشى و طراحى مطالعه: حسين همتى، سيده

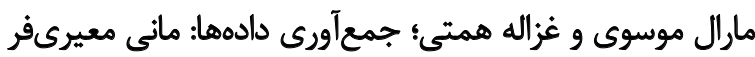

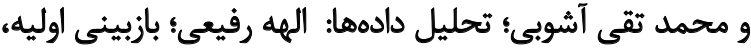
تأييد نسخه نهايى: همه نويسندكان.

$$
\text { تمارض مثانع }
$$

بنابر اظهار نويسندكان اين مقاله تعارض منافع ندارد.

$$
\text { تشكر و قدروانى ته }
$$

نويسندكان اين مقاله از دانشكاه علوم يزشكى كيلان وإن وإيند

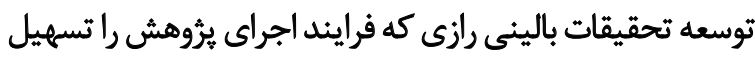
نموده اند، تشكر و سياسكزارى مي كيندا 


\section{References}

[1] Youn YJ, Lee J. Chronic venous insufficiency and varicose veins of the lower extremities. The Korean Journal of Internal Medicine. 2019; 34(2):269-83. [DOI:10.3904/kjim.2018.230] [PMID]

[2] Yetkin E, Ozturk S. Dilating vascular diseases: Pathophysiology and clinical aspects. International Journal of Vascular Medicine. 2018; 2018:9024278. [DOI:10.1155/2018/9024278] [PMID]

[3] Sam RC, Silverman SH, Bradbury AW. Nerve injuries and varicose vein surgery. European Journal of Vascular and Endovascular Surgery: The Official Journal of the European Society for Vascular Surgery. 2004; 27(2):113-20. [DOI:10.1016/j.ejvs.2003.11.007] [PMID]

[4] O'Hare JL, Vandenbroeck CP, Whitman B, Campbell B, Heather BP, Earnshaw JJ, et al. A prospective evaluation of the outcome after small saphenous varicose vein surgery with one-year follow-up. Journal of Vascular Surgery. 2008; 48(3):669-73. [DOI:10.1016/j.jvs.2008.04.041] [PMID]

[5] Allegra C, Antignani PL, Carlizza A. Recurrent varicose veins following surgical treatment: Our experience with five years follow-up. European Journal of Vascular and Endovascular Surgery: The Official Journal of the European Society for Vascular Surgery. 2007; 33(6):751-6. [DOI:10.1016/j.ejvs.2006.12.020] [PMID]

[6] Nijsten T, van den Bos RR, Goldman MP, Kockaert MA, Proebstle TM, Rabe $E$, et al. Minimally invasive techniques in the treatment of saphenous varicose veins. Journal of the American Academy of Dermatology. 2009; 60(1):110-9. [DOI:10.1016/j.jaad.2008.07.046] [PMID]

[7] Smith PC. Chronic venous disease treated by ultrasound guided foam sclerotherapy. European Journal of Vascular and Endovascular Surgery. 2006; 32(5):577-83. [DOI:10.1016/j.ejvs.2006.04.033] [PMID]

[8] Whiddon LL. The treatment of venous ulcers of the lower extremities. Baylor University Medical Center Proceedings. 2007; 20(4):363-6. [DOI: 10.1080/08998280.2007.11928324] [PMID]

[9] Sarma N. Guidelines and recommendation on surgery for venous incompetence and leg ulcer. Indian Dermatology Online Journal. 2014; 5(3):390-5. [DOI:10.4103/2229-5178.137825] [PMID]

[10] Kurihara N, Hirokawa M, Yamamoto T. Postoperative venous thromboembolism in patients undergoing endovenous laser and radiofrequency ablation of the saphenous vein. Annals of Vascular Diseases. 2016; 9(4):259-66. [DOI:10.3400/avd.oa.16-00087] [PMID]

[11] Rautio T, Ohinmaa A, Perälä J, Ohtonen P, Heikkinen T, Wiik H, et al. Endovenous obliteration versus conventional stripping operation in the treatment of primary varicose veins: A randomized controlled trial with comparison of the costs. Journal of Vascular Surgery. 2002; 35(5):95865. [DOI:10.1067/mva.2002.123096] [PMID]

[12] Lurie F, Creton D, Eklof B, Kabnick LS, Kistner RL, Pichot O, et al. Prospective randomized study of endovenous radiofrequency obliteration (closure procedure) versus ligation and stripping in a selected patient population (EVOLVeS Study). Journal of Vascular Surgery. 2003; 38(2):207-14. [DOI:10.1016/S0741-5214(03)00228-3] [PMID]

[13] Sincos IR, Baptista APW, Coelho Neto F, Labropoulos N, Alledi LB, de Marins EM, et al. [Prospective randomized trial comparing radiofrequency ablation and complete saphenous vein stripping in patients with mild to moderate chronic venous disease with a 3-year follow-up (English-Portuguese)]. Einstein. 2019; 17(2):eAO4526. [DOI:10.31744/ einstein_journal/2019AO4526] [PMID] [PMCID]

[14] Almeida JI, Kaufman J, Gockeritz O, Chopra P, Evans MT, Hoheim DF, et al. Radiofrequency endovenous ClosureFAST versus laser ablation for the treatment of great saphenous reflux: A multicenter, single-blinded, randomized study (RECOVERY study). Journal of Vascular and Interventional Radiology. 2009; 20(6):752-9. [DOI:10.1016/j.jvir.2009.03.008]

[15] Ravi R, Rodriguez-Lopez JA, Trayler EA, Barrett DA, Ramaiah V, Diethrich $E B$. Endovenous ablation of incompetent saphenous veins: $A$ large single-center experience. Journal of Endovascular Therapy. 2006; 13(2):244-8. [DOI:10.1583/05-1760R.1] [PMID]

[16] Padberg Jr FT. CEAP classification for chronic venous disease. Disease-aMonth. 2005; 51(2-3):176-82. [DOI:10.1016/j.disamonth.2005.03.013] [PMID]

[17] Vasquez MA, Rabe E, McLafferty RB, Shortell CK, Marston WA, Gillespie $D$, et al. Revision of the venous clinical severity score: Venous outcomes consensus statement: Special communication of the American Venous Forum Ad Hoc Outcomes Working Group. Journal of Vascular Surgery. 2010; 52(5):1387-96. [DOI:10.1016/j.jvs.2010.06.161] [PMID]

[18] Monahan TS, Belek K, Sarkar R. Results of radiofrequency ablation of the small saphenous vein in the supine position. Vascular and Endovascular Surgery. 2012; 46(1):40-4. [DOI:10.1177/1538574411425108] [PMID]

[19] Theivacumar NS, Beale RJ, Mavor AID, Gough MJ. Initial experience in Endovenous Laser Ablation (EVLA) of varicose veins due to small saphenous vein reflux. European Journal of Vascular and Endovascular Surgery. 2007; 33(5):614-8. [DOI:10.1016/j.ejvs.2006.10.030] [PMID]

[20] Choi JH, Park HC, Joh JH. The occlusion rate and patterns of saphenous vein after radiofrequency ablation. Journal of the Korean Surgical Society. 2013; 84(2):107-13. [DOI:10.4174/jkss.2013.84.2.107] [PMID]

[21] Samuel N, Carradice D, Wallace T, Mekako A, Hatfield J, Chetter I. Randomized clinical trial of endovenous laser ablation versus conventional surgery for small saphenous varicose veins. Annals of Surgery. 2013; 257(3):419-26. [DOI:10.1097/SLA.0b013e318275f4e4] [PMID]

[22] Doganci S, Yildirim V, Demirkilic U. Does puncture site affect the rate of nerve injuries following Endovenous laser ablation of the small saphenous veins? European Journal of Vascular and Endovascular Surgery. 2011; 41(3):400-5. [DOI:10.1016/j.ejvs.2010.11.029] [PMID]

[23] van Groenendael L, Flinkenflögel $L$, van der Vliet JA, Roovers EA, van Sterkenburg SM, Reijnen MM. Conventional surgery and Endovenous laser ablation of recurrent varicose veins of the small saphenous vein: A retrospective clinical comparison and assessment of patient satisfaction. Phlebology. 2010; 25(3):151-7. [DOI:10.1258/phleb.2009.009044] [PMID]

[24] Trip-Hoving M, Verheul JC, van Sterkenburg SM, de Vries WR, Reijnen $\mathrm{MM}$. Endovenous laser therapy of the small saphenous vein: Patient satisfaction and short-term results. Photomedicine and Laser Surgery. 2009; 27(4):655-8. [DOI:10.1089/pho.2008.2389] [PMID]

[25] Boersma D, Kornmann VN, van Eekeren RR, Tromp E, Ünlü Ç, Reijnen $\mathrm{MM}$, et al. Treatment modalities for small saphenous vein insufficiency: Systematic review and meta-analysis. Journal of Endovascular Therapy. 2016; 23(1):199-211. [DOI:10.1177/1526602815616375] [PMID]

[26] Harlander-Locke M, Jimenez JC, Lawrence PF, Derubertis BG, Rigberg DA, Gelabert HA, et al. Management of endovenous heat-induced thrombus using a classification system and treatment algorithm following segmental thermal ablation of the small saphenous vein. Journal of Vascular Surgery. 2013; 58(2):427-31. [DOI:10.1016/j.jvs.2013.01.026] [PMID]

[27] Janne d'Othée B, Walker TG, Kalva SP, Ganguli S, Davison B. Endovenous laser ablation of the small saphenous vein sparing the saphenopopliteal junction. Cardiovascular and Interventional Radiology. 2010; 33(4):766-71. [DOI:10.1007/s00270-010-9806-1] [PMID] 
[28] Carradice D, Samuel N, Wallace T, Mazari FA, Hatfield J, Chetter I. Comparing the treatment response of great saphenous and small saphenous vein incompetence following surgery and endovenous laser ablation: $A$ retrospective cohort study. Phlebology: The Journal of Venous Disease. 2012; 27(3):128-34. [DOI:10.1258/phleb.2011.011014] [PMID]

[29] Desmyttère J, Grard C, Stalnikiewicz G, Wassmer B, Mordon S. Endovenous laser ablation $(980 \mathrm{~nm})$ of the small saphenous vein in a series of 147 limbs with a 3-year follow-up. European Journal of Vascular and Endovascular Surgery. 2010; 39(1):99-103. [DOI:10.1016/j. ejvs.2009.09.011] [PMID]

[30] Lawrence PF, Chandra A, Wu M, Rigberg D, DeRubertis B, Gelabert $\mathrm{H}$, et al. Classification of proximal endovenous closure levels and treatment algorithm. Journal of Vascular Surgery. 2010; 52(2):388-93. [DOI:10.1016/j.jvs.2010.02.263] [PMID]

[31] Marsh P, Price BA, Holdstock J, Harrison C, Whiteley MS. Deep Vein Thrombosis (DVT) after venous thermoablation techniques: Rates of Endovenous Heat-Induced Thrombosis (EHIT) and classical DVT after radiofrequency and endovenous laser ablation in a single centre. European Journal of Vascular and Endovascular Surgery. 2010; 40(4):521-7. [DOI:10.1016/j.ejvs.2010.05.011] [PMID] 
This Page Intentionally Left Blank 